\title{
The Soweto Stroke Questionnaire
}

\begin{abstract}
A questionnaire was designed for a recent survey into the outcome of stroke patients in Soweto, named the Soweto Stroke Questionnaire (SSQ). It LA HALE CJ EALES VU FRITZ was based on the Barthel ADL Index (BI) but modified to suit the local context. This paper introduces the SSQ, and reports on its inter-rater reliability and its concurrent validity. Fifty-four subjects, in the age range 30 to 75 years, were interviewed and nineteen re-interviewed using the SSQ. Four different scores were calculated: a total score, a Barthel Index score, an Impairment score, and a Quality of Life score. The Pearson's Correlation Coefficient was found to be high between the total score and the BI score. $(r=0.948)$ which supports the concurrent validity of the developed questionnaire. In assessing the reliability of the $S Q Q$, the Wilcoxin Test showed that there was no significant difference between the initial and repeat interviews for the total score, the Barthel Index score, and the Impairment score $(p<0,05)$. The Quality of Life Score came closer to a difference, but not statistically significantly so. These tests were collaborated by Bland and Altman graphs which showed that in $95 \%$ of the time, the questions were repeatable. Mc Nemar's Test of Symmetry showed that 34 out of 38 questions asked were found to have over $70 \%$ correlation. Four questions showed a lower correlation, the lowest being 63.16\%. The SSQ was found to have interrater reliability, and to be concurrently valid to the Barthel Index. It is quick and easy to use, requiring no sophisticated equipment or training. It still requires to be investigated for sensitivity and predictiveness, and to be validated in a more general South African stroke population.
\end{abstract}

\section{KEY WORDS: STROKE OUTCOME, BARTHEL INDEX, SOUTH AFRICA}

Although stroke is ranked as the second most common cause of death in South Africa its prevalence in South Africa is not well known (Fritz, 1997). The Central Statistical Services (1980) reported that cerebrovascular accident accounted for 298/100,000 deaths in Coloured South Africans aged 35-74 years, and $100 / 100,000$ deaths in the White population. The statistical data for the Black population of South Africa is imprecise (Fritz 1997). Putteril et al (1984) predicted that the incidence of non-fatal stroke in South Africans over 75 years of age was likely to increase from $100-200 / 100,000$ to $2000 / 100,000$.

A review of the literature revealed numerous stroke outcome measuring scales. Some scales predominantly measure impairment and motor recovery (Carr et al, 1972; Gowland et al, 1993). Disability measures of basic activities of daily living (ADL) (attention

\section{CORRESPONDENCE:}

Mrs L A Hale

Physiotherapy Department

Faculty of Health Sciences

University of the Witwatersrand

7 York Road, Parktown, 2193, South Africa to hygiene, dressing, transfers, ambulation, continence and feeding) include the Barthel Index (Collin et al, 1988) and the Katz Index of ADL (Brorsson and Asberg, 1984). Instrumental ADL, for example the Functional Independence Measure (FIM) (Hamilton et al, 1994; Glott, 1995), explores higher degrees of ADL such as domestic chores, transportation and communication. The FIM is gaining popularity in South Africa. However, it requires appropriate training and trainer testing before a high level of reliability can be achieved (Hamilton et al, 1994). Data can be converted from ordinal ratings to a linear scale with interval properties using Rasch analysis. This data can be plugged into a data bank built up of over 100000 FIM ratings in order to predict management, length of hospital stay and outcome (Glott, 1995). However, as the FIM scale appears not to have been validated in South Africa, this data prediction facility is invalid as one cannot equate data collected in the United States of America to the South African context.

Measuring handicap is a lot more complex as it has to take into account the many individual characteristics that affect an individual's social role fulfilment, for example, education, family support, financial resources, as well as dealing with societal limitations (Whiteneck, 1994). The CIQ (Community Integrated Questionnaire) designed by Willer et al (1994) is an example of a scale which measures handicap. The CIQ was developed for persons with traumatic head-injury and deals primarily with instrumental ADL and handicap. Although it measures the success of outcome, namely, social integration, it gives little assistance in the planning of an intervention (Willer et al, 1994). In order for a rehabilitation measurement scale to be versatile and efficient, it needs to measure impairment (in order to plan the intervention), disability and handicap (to measure the outcome of the intervention).

Dr Derek Wade of the Rivermead Rehabilitation Centre in Oxford suggests that one of the best "buys" on the market is the Barthel lndex (Collin et al, 1988). It is has been used extensively in stroke outcome studies. This ordinal scale measures basic functional outcome, requires very little training and has been found to be both reliable and valid for stroke (Wade and Hewer, 1987). However, it's limitations include the fact that it only measures disability. The BI has not, to the best of my knowledge, been validated in South Africa; it is unable to measure 
quality of recovery; and has been found to be insensitive in the higher scores with a low ceiling of measure (Smith, 1993).

The Soweto Stroke Questionnaire was designed for a recent survey into the problems facing Sowetan stroke patients post-discharge from Chris Hani (CH) Baragwanath Hospital and may prove to be a basis from which to design a more specific South African measuring scale.
The aim of this paper is to introduce the Soweto Stroke Questionnaire, and to report on its inter-rater reliability and its concurrent validity.

\section{THE SOWETO STROKE QUESTIONNAIRE}

Although the questionnaire has both qualitative and quantitative aspects (see Table 1), only the quantitative side will be dealt with in this paper. The quantitative section is

TABLE 1: SOWETO STROKE QUESTIONNAIRE

QUANTITATIVE

Total score (50)

Home

Circumstances

(3)

mpairment

Score

(8)
QUALITATIVE

(coded)

Quality of Life Score

(8)
Expanded

Barthel Index

(31) (Embedded BI = 20)

TABLE 2: CORRELATION OF TOTAL SCORES $(n=19)$

$\begin{array}{lll} & \text { Total Score - Initial (50) } & \text { Total Score - Repeat (50) } \\ \text { Mean } & 25.95 & 26.42 \\ \text { Std. Dev } & +/-14.41 & +/-13.10 \\ \text { Range } & 5-46 & 4-47 \\ \text { WILCOXIN } & 0.93 & \end{array}$

TABLE 3: CORRELATION OF IMPAIRMENT SCORES $(n=19$ )

$\begin{array}{lll} & \text { Total Score - Initial (8) } & \text { Total Score - Repeat (8) } \\ \text { Mean } & 4.05 & 4.16 \\ \text { Std. Dev } & +/-2.29 & +/-2.36 \\ \text { Range } & 1-8 & 0-8 \\ \text { WILCOXIN } & \mathbf{0 . 8 7} & \end{array}$

TABLE 4: CORRELATION OF QUALITY OF LIFE SCORES $(n=19)$

$\begin{array}{lll} & \text { Total Score }- \text { Initial (8) } & \text { Total Score - Repeat (8) } \\ \text { Mean } & 3.16 & 2.68 \\ \text { Std. Dev } & +/-1.92 & +/-1.73 \\ \text { Range } & 0-7 & 0-7 \\ \text { WILCOXIN } & \mathbf{0 . 0 8 4} & \end{array}$

TABLE 5: CORRELATION OF BARTHEL INDEX SCORES $(n=19)$

$\begin{array}{lll} & \text { Total Score }- \text { Initial }(20) & \text { Total Score - Repeat (20) } \\ \text { Mean } & 12.26 & 12.68 \\ \text { Std. Dev } & +/-7.22 & +/-6.44 \\ \text { Range } & 0-20 & 0-20 \\ \text { WILCOXIN } & 0.86 & \end{array}$

scored on an ordinal scale, giving a total score of fifty points. This total score can then be subdivided into four sections, there being no weighting to the distribution of the scoring (see Table 1):

1. Data relating to the patients' home circumstances: whether their toilet is located inside the house or outside; do they have running water and electricity in the home. This data was deemed necessary to collect so that the context of the home could be invisualised.

2. Data relating to the known complications of stroke, for example, pain in the hemiplegic shoulder and knee; pressures sores and contactures. This is known as the Impairment Score. Muscle tone, strength and sensory status were not measured.

3. Data relating to the patient's disability - the Barthel Index (BI) is incorporated into the questionnaire, and is expanded in order to gain more insight regarding functional ability, the BI being shown to be insensitive in the higher scores known as the expanded Barthel Index score.

4. Data relating to handicap: ability to help with household chores, socialisation, outings, ability to catch a minibus taxi - known as the Quality of Life score.

\section{INTER-RATER RELIABILITY AND CONCURRENT VAIIDITY}

\section{METHODOLOGY}

All cerebrovascular accident (CVA) patients admitted to the medical admissions ward of $\mathrm{CH}$ Baragwanath Hospital over a four and a half month period were screened for admission into the study. The inclusion criteria of the study required that patients, of either sex, who had their first and only CVA in the region of the middle cerebral artery, and who resided in Soweto be included. Patients had to be in the age range 30 to 75 years. The patients' names were acquired from the ward's admission register, and information regarding their inclusion suitability obtained from their medical files.

The selected subjects were then interviewed in their homes three 
months post-discharge from hospital using the Soweto Stroke Questionnaire (SSQ), after informed consent had been obtained from each subject. The interviews were conducted by physiotherapy assistants trained in the use of the SSQ. Subjects were then randomly chosen by an "out-the-hat" method for repeat interviews. These repeat interviews were performed by different interviewers in order to evaluate inter-rater reliability.

\section{RESIJLTS}

A total of 361 subjects were admitted during the selection period. Ninety-three patients died and 214 did not fulfill the selection criteria. Fifty-four subjects comprised the final study group who were interviewed in their homes. Nineteen subjects were interviewed twice within a period of sixty days. The following scores were calculated from each interview: Total score, Impairment score, Quality of Life score, and a Barthel Index score.

In order to test inter-rater reliability, the above mentioned scores of the initial interviews were correlated with those of the repeat interviews using the Wilcoxin test (see Tables 2, 3, 4, 5). A high degree of correlation was shown in the case of the Total scores, the Impairment scores and the Barthel Index scores $(p<0,05)$, indicating that there was a degree of correlation for these scores between the initial interview and the repeat interview.

The Quality of Life Score, did not show a statistically significant difference between the initial and repeat interview mean scores, and its degree of correlation was not as high as with the other scores.

The SSQ was then tested for the degree of reliability question for question using Mc Nemar's test for symmetry (Table 6). This test showed a greater than $70 \%$ correlation between the initial and repeat interviews for 34 out of 38 questions asked. Four questions showed a lower correlation, the lowest being $63 \%$.

These results were collaborated by Bland and Altman graphs which showed that in $95 \%$ of the time, the SSQ questions were repeatable.

In order to show concurrent validity the Total score of the SSQ was correlated to the BI scores using Pearson's correlation co-efficient (Table 7), bearing in mind that the BI has already been shown to have reliability and validity internationally. The Pearson's Correlation Coefficient was found to be high between the total score and the $\mathrm{BI}$ score $(r=0.948)$.

\section{DISCUSSION}

The criteria for a South African outcome measuring tool has already been outlined. In many respects, the SSQ has been shown to fulfill these requirements.

Reliability has been established at the inter-rater level for the Total scores, Impairment scores, and the expanded Barthel Index score. This reliability was less evident in the Quality of Life score. This is not surprising as "Quality of Life" is a dynamic construct, and can not be expected to be the same at different times during a disease process. The reduced correlation may be due to the initial support offered by the subject's friends and family which may have waned as time passed and the interest grew less, as often is the case. The initial interviews may have been conducted while the social support system was still good, and the repeat interviews performed after this phase was over. Question for question, the SSQ showed a high degree of reliability. The physiotherapy assistants had logistical difficulties in revisiting subjects. For example, the lack of telephones prevented appointments from being set-up; when arriving at a home, the subjects had often being locked into their homes by their relatives for their own safety; the assistants often had to travel far and with many taxis in order to reach some of the homes. These are real problems, and as stroke is a chronic disorder, unlikely to change rapidly with time, the time periods used in the study were felt not to be unrealistic.

The Barthel Index has been shown in the past to have both reliability and validity. The SSQ is based on the BI, expanded with additional questions felt to be relevant for a specific research study. The SSQ was found to be highly correlated to the $\mathrm{BI}$, indicating that the additional questions did not alter the validity of the Index.

Furthermore, the SSQ demonstrates versatility in that it provides both a measure of functional outcome and information on which to plan intervention: it measures impairment, disability and handicap. In a country where resources are limited, a tool which measures more than one thing in a simple manner is a very valuable commodity.

Two important criteria were that the tool should be quick and simple to administer, and not require sophisticated equipment. Physiotherapy assistants were taught how to use the SSQ in two training sessions, and were able to conduct the interview in less than an hour, with no problems. The assistants were all residents of Soweto, and when asked their opinion regarding which languages the questionnaire should be translated into (remembering that South Africa has eleven official languages), they were
TABLE 6: Mc NEMAR'S TEST OF SYMMETRY $(n=19)$

(Question for question correlation between initial and repeat interviews)

$\begin{array}{ll}\% \text { Correlation } & \text { Number of Questions } \\ 100 \% & 5 \\ 90-99 \% & 5 \\ 80-89 \% & 12 \\ 70-79 \% & 12 \\ 60-69 \% & 4 \\ <60 \% & 0\end{array}$


adamant that it should be in English. The only equipment required was the questionnaire and a pen.

The final criteria was that the SSQ should have credibility in the international community. This has been achieved by having the Barthel Index embedded within the SSQ.

Still to be shown is the test-retest reliability of the scale; and its content, construct and predictive validity. It must be validated to the South African stroke population in general. The time taken to administer the questionnaire and its responsiveness must still be specifically established.

So where to from here? If all that is required is a functional baseline measurement, then the Barthel Index is probably the quickest and easiest method to use.

However, the Barthel Index has been criticised for not having the ability to measure the quality of the recovery of functional movement, and in the higher scores has been found to be insensitive in discerning the degree of recovery (Smith, 1993). The highest functional levels assessed are whether the subject can walk independently (with or without an appliance) and manage stairs. Walking in different environmental conditions is not ascertained. This often requires a greater degree of recovery than walking indoors.

The Barthel Index has been widely used in the United Kingdom but its suitability for the South African stroke population has not been investigated, and needs to be established. For example, the ability to go to the toilet independently is a func- tion measured on the Barthel Index. In Soweto many houses have outside toilets accessed by dirt paths. This requires a greater degree of ability to manage independently. Stairs are often not encountered by residents of Soweto who are confined to their single-storied homes and surroundings, and therefore this question is of doubtful value. The ability to wash one's body may require a greater degree of ability if one has to fetch one's water from a community source and then use it standing in a tin basin. Therefore a subject in Soweto with the same Barthel Score as a subject in England could actually be functioning from a different disability level. Within a given population group of similar home conditions the scoring will be standard, unfortunately these conditions are not always met in Soweto, as people live under different conditions, from squatter camps to fair sized houses with inside bathroom facilities.

The Barthel Index only measures disability. This makes its usefulness in designing a rehabilitation programme limited as the reasons (the impairments) for the disability are not identified. In rehabilitation these reasons are addressed in order to improve the degree of disability. Improvement in disability, hopefully but not necessarily, will then translate into an improvement of handicap or quality of lifestyle.

The SSQ probably provides more information on which to base management and intervention than the BI. It can thus be argued that its construct validity has been demonstrated. However, content and

\section{TABLE 7: CORRELATION OF TOTAL SCORE TO BARTHEL INDEX SCORES ( $n=54)$}

$\begin{array}{lll} & \text { Initial (Total Score = 50) } & \text { Repeat (Total Score = 20) } \\ \text { Mean } & 31.73 & 15.21 \\ \text { Std. Dev } & +/-12.49 & +/-5.84 \\ \text { Median } & 34 & 18 \\ \text { Range } & 5-48 & 0-20 \\ \begin{array}{l}\text { Pearson's } \\ \text { Correlation } \\ \text { Coefficient }\end{array} & 0.95 & \end{array}$

predictive validity, and responsiveness still need to be established. These items are presently being researched both in the Sowetan community with an upgraded questionnaire, as well as in a rural setting of South Africa.

The SSQ is an ordinal scale in that it hierarchally classifies stroke patients. It has been argued that ordinal scales only give a relative, and not a definite, measure, and thus should not be added to a sumscore unless specifically justified (Sødring, 1995). However, the SSQ is predominantly a "yes/no" scale. Thus it exhibits ratio-scale properties: there are only two possible states. These can be represented statistically as " 0,1 " and can thus be used for ANOVAs or correlations (Krebs, 1987). Dichotomous scales show, on the one hand, high reliability, however, on the other hand, the lack of choice can often decrease their sensitivity (Sødring, 1995). Embedded within the SSQ, is the Barthel Index. The Barthel Index, also an ordinal scale, has been added to sumscores which have frequently been used in statistical analysis. Therefore, statistical analysis of the SSQ data for research is justifiable.

The scoring of the SSQ carries no weighting, for example, the impairment and quality of life subsections are scored out of eight, whereas ADL is scored out of twenty. This by no means reflects a perceived importance of one subsection over another. Further investigation into the scoring is required.

\section{CONCLUSION}

The SSQ is in its infancy, however it does provide both a functional status and an impairment measure. Although it was designed to meet the criteria of a specific research study, it does fulfill the requirements for an outcome measuring scale suitable for South Africa.

Jules Rothstein, editor of Physical Therapy recently wrote in an editorial entitled "Break on Through": "Accountability, evidence-based practice, and out-come based management are not ploys of an evil empire, just concepts that are difficult to implement - and almost 
impossible to logically argue against" (Rothstein, 1997).

It is imperative that stroke rehabilitation in South Africa be measured, and thus an appropriate tool is highly desirable.

\section{REFERENCES}

Brorsson B, Asberg KH. Katz Index of independence in ADL: reliability and validity in short-term care. Scand J Rehabil Med 1984: 16: $125-132$

Carr JH, Shepherd RB, Nordholm L, Lynne D. Investigation of a new motor assessment scale for stroke patients. Phys Ther 1972; 65: $175-178$

Central Statistical Services. Report on Deaths: Whites, Coloureds and Asians, 1980: Report 07-03-19. Government Printer, Pretoria

Collin C, Wade DT, Davies S, Horne V. The Barthel Index: a reliability study. Int. Disabil. Studies 1988; 10 (2): 61 - 63
Fritz VU. Stroke incidence in South Africa. S Afr Med J 1997; 87(5): 584 - 585

Glott T. Functional independence measure scale on stroke patients. Physiotherapy in Stroke Management. Edited by M. A. Harrison, Churchill Livingstone, Edinburgh, 1995, Chapter 16, 139 - 144

Gowland C, Stratford P, Ward M, et al. Measuring physical impairment and disability with the Chedoke-Mc Master stroke assessment. Stroke 1993; 24 (1): 58 - 63

Hamilton BB, Laughlin JA, Roger CF, Granger CV. Inter-rater reliability of the seven level Functional Independence Measure (FIM). Scand J Rehabil Med 1994; 26: 115 - 119

Krebs DE. Measurement theory. Phys Ther 1987; 67 (12): 1834 - 1839

Putteril JS, Disler PB, Jacka E. Coping with chronic illness. Part II. Cerebrovascular accidents. S Afr Med J 1984; 65: 891 - 893.

Rothstein JM. Break on through (Editorial) . Physical Therapy 1997; 77 (12): 1680
Smith A. Beware of the Barthel. Physiotherapy 1993; 79 (12): 843 - 844

Sødring KM. The Sødring motor evaluation of stroke patients. Physiotherapy in Stroke Management. Edited by M.A. Harrison, Churchill Livingstone, Edinburgh, 1995, Chapter 14, 119 - 123

Wade DT, Langton-Hewer R. Functional abilities after stroke measurement, natural history and prognosis. J Neuro Neurosurg Pysch 1987; 50: 177 - 182

Whiteneck GG. Measuring what matters: key rehabilitation outcomes. The 44th annual John Stanley Coulter lecture. Arch Phys Med Rehabil 1994; 75: 1073 - 1076

Willer B, Ottenbacher KJ, Coad ML. The community intergration questionnaire. Am J Phys Med Rehabil 1994: 73 (2): 103 - 111

This study was granted ethical clearance from the Committee for Research on Human Subjects, University of the Witwatersrand: No. 950111 . The study was funded by the Medical Research Council of South Africa.

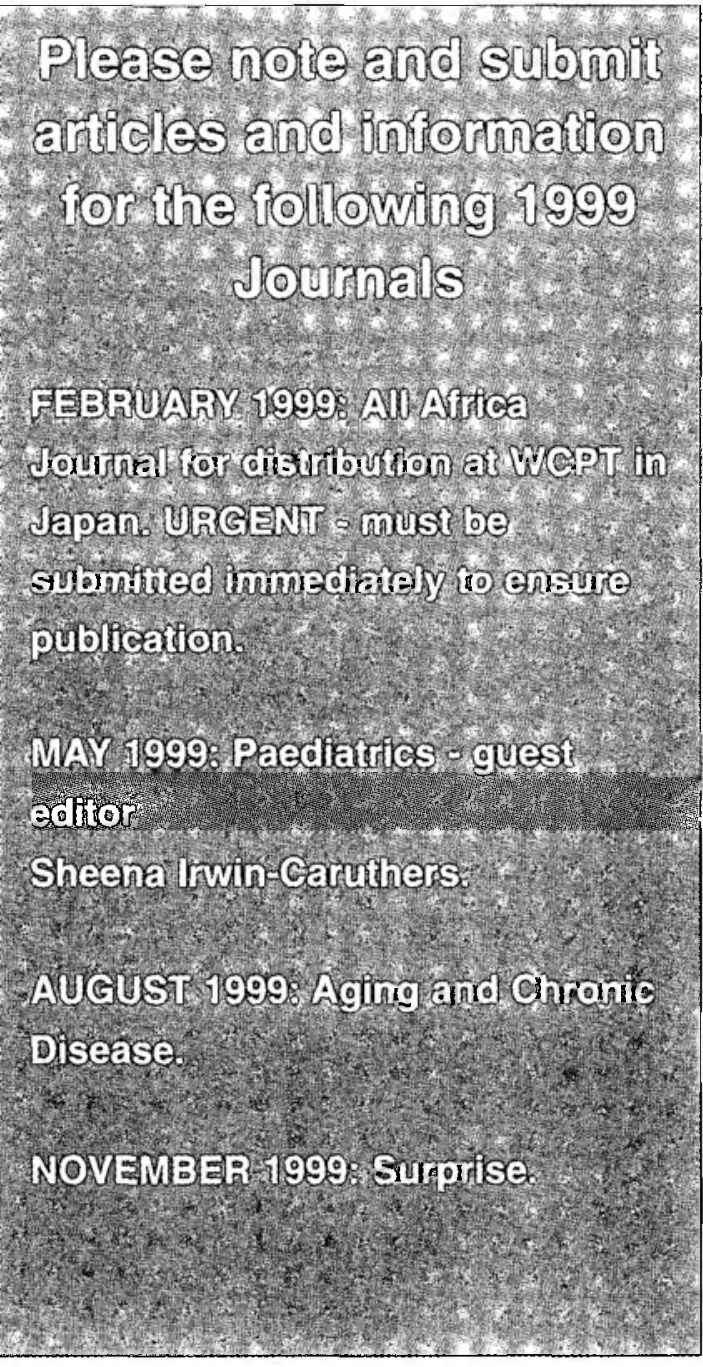

\section{The College of Physiotherapists of South Africa (CPSA)}

The CPSA is an independent examining body for clinical specialisation. It is currently also in the process of developing an accreditation and certification system for Continuing Physiotherapy Education (CPE) courses. Certificates issued by a body such as the CPSA have more national and international recognition than certificates issued by a Special Interest Group or private companies.

Any one running or organising $\mathrm{CPE}$ courses can apply for accreditation of such courses. It is envisaged that the process of accreditation and certification will commence early in 1999.

For further information please contact: The Registrar or President of the CPSA, Physiotherapy Department, Medical School, 7 York Road, Parktown, 2193 Johannesburg. 